\title{
EMBEDDING STIELTJES-VOLTERRA INTEGRAL EQUATIONS IN STIELTJES INTEGRAL EQUATIONS( ${ }^{(1)}$
}

BY

\author{
WILLIAM L. GIBSON
}

\begin{abstract}
J. A. Reneke has shown that the linear Stieltjes-Volterra integral equations studied by D. B. Hinton can be transformed into Stieltjes integral equations of the type studied by J. S. Mac Nerney. By taking advantage of the nonlinear nature of Mac Nerney's results, Reneke was able to extend Hinton's existence theorem to a nonlinear setting. In this paper, we use Reneke's embedding technique to generalize several other of Hinton's results, and we characterize completely, in the linear case, the range of Reneke's embedding transformation.
\end{abstract}

Introduction. In [7] J. A. Reneke showed that part of D. B. Hinton's theory of Stieltjes-Volterra integral equations with its associated transformation $\mathcal{X}$ [1] could be subsumed in J. S. Mac Nerney's theory of Stieltjes integral equations with its associated transformation $\&$ [6]. Reneke accomplished this by introducing a transformation $\Re$ which embeds Hinton's class $F$ of kernel functions into Mac Nerney's class $O A$ of kernel functions in such a way that for each of Hinton's Stieltjes-Volterra integral equations, there arises a Stieltjes integral equation from the solution of which a solution to the original equation may be obtained. By taking advantage of the nonlinear nature of $\mathscr{E}$, Reneke extended Hinton's existence theorem to a nonlinear setting.

In this paper we study Reneke's technique and introduce certain refinements which clarify and extend Reneke's results. Specifically, we show (Theorem 2.1) that each member of the range of $R$ has a basic substitution property which permits the use of a nonlinear integration by substitution process (Theorem 2.2 ) through which one can quickly obtain a generalization (Theorem 2.3) of Reneke's main result. Restricting our attention to the set $F^{\prime}$

Presented to the Society, November 23, 1974 under the title $A$ correspondence associated with Stieltjes-Volterra integral equations; received by the editors November 13, 1975.

AMS (MOS) subject classifications (1970). Primary 45D05, 45G99, 45N05; Secondary 28A25, 28A40, 34A10, 26A39.

Key words and phrases. Stieltjes-Volterra integral equations, nonlinear integration by substitution, hereditary system, quasicontinuous functions.

(') This paper extends parts of the author's dissertation, done under the direction of J. S. Mac Nerney at the University of Houston, and partly supported by NASA Grant NGL 44-005-090. 
of "linear" members of $F$ allows us to duplicate the setting in which Hinton worked, and we obtain a generalization (Theorem 2.4) of Hinton's theorem which represents the solution of the nonhomogeneous equation in terms of the solution of the homogeneous equation. Next, a new proof (Theorem 2.5) of Hinton's main result is given, showing that his transformation is its own inverse.

Our last section gives a complete characterization (Theorem 3.2) of the sets $\Re\left(F^{\prime}\right)$ and $\mathcal{E}\left(\Re\left(F^{\prime}\right)\right)$ and draws a connection (Theorem 3.3) between this and the input-output structure for hereditary-type systems associated with linear Stieltjes-Volterra integral equations.

1. Preliminaries. Suppose $S$ denotes a number interval $[a, b]$ and $O$ denotes the usual $\leqslant$ ordering for $S$. As in [3], let $O A^{+}$denote the class of all $O$-additive functions from $S \times S$ into the set of all nonnegative real numbers, and $\mathrm{OM}^{+}$ denote the class of all $O$-multiplicative functions from $S \times S$ into the set of all real numbers not less than 1 . There exists [3, Theorem 2.2] a reversible function $\mathcal{E}^{+}$from $\mathrm{OA}^{+}$onto $O \mathrm{M}^{+}$to which the ordered pair $\{a, m\}$ belongs only in case one of the following holds:

(a) $a$ is in $O A^{+}$and $m(x, y)={ }_{x} \Pi^{y}[1+a]$ for all $\{x, y\}$ in $S \times S$.

(b) $m$ is in $O M^{+}$and $a(x, y)={ }_{x} \Sigma^{y}[m-1]$ for all $\{x, y\}$ in $S \times S$.

Let $\left\{G_{1}, N_{1}\right\}$ denote a normed and complete Abelian group, let $H_{1}$ denote the set of all functions from $G_{1}$ into $G_{1}$ which contain the ordered pair $\{0,0\}$, and let $H_{1}^{\prime}$ denote the set of all members $A$ of $H_{1}$ which are additive; i.e. $A(P+Q)=A(P)+A(Q)$ for all $\{P, Q\}$ in $G_{1} \times G_{1}$.

At a higher level, there is another group structure which was introduced by Reneke [7, p. 623] as follows: Let $\left\{G_{2}, N_{2}\right\}$ denote the normed complete group of all quasicontinuous functions from $\{S, O\}$ into $\left\{G_{1}, N_{1}\right\}$ normed with the supremum (relative to $N_{1}$ ) norm $N_{2}$. The zero of $G_{2}$ is the member of $G_{2}$ whose only value is the zero of $G_{1}$, and addition in $G_{2}$ is functional addition. $H_{2}$ denotes the set of all functions from $G_{2}$ into $G_{2}$ which contain $\{0,0\}$, and $H_{2}^{\prime}$ denotes the set of all additive members of $H_{2}$. Let $O A$ and $O M$ denote J. S. Mac Nerney's classes [6, p. 622] associated with the system $\left\{S, O, G_{2}, N_{2}, H_{2}\right\}$.

Mac Nerney's transformation $\mathcal{E}$. There exists [6, Theorem 1.1] a reversible transformation $\mathscr{E}$ from $O A$ onto $O M$ such that each of the following is a necessary and sufficient condition for the member $\{V, W\}$ of $O A \times O M$ to belong to $\varepsilon$.

(a) $W$ is in $O M$ and $V(x, y) g={ }_{x} \Sigma^{y}[W-1] g$ for each $\{x, y, g\}$ in $S \times S$ $\times G_{2}$.

(b) $V$ is in $O A$ and $W(x, y) g={ }_{x} \Pi^{y}[1+V] g$ for each $\{x, y, g\}$ in $S \times S$ $\times G_{2}$.

(c) There is an ordered pair $\{a, m\}$ in $\mathcal{E}^{+}$such that if $\{x, y\}$ is in $S \times S$ and $g$ is in $G_{2}$, then 


$$
N_{2}([W(x, y)-1-V(x, y)] g) \leqslant\{m(x, y)-1-a(x, y)\} N_{2}(g) .
$$

The Stieltjes right-integral equation. Mac Nerney shows [6, Corollary 2.1] that if $\{V, W\}$ is in $\mathcal{E}$, then

$$
W(x, y) g=g+(\mathrm{R}) \int_{x}^{y} V W[I, y] g
$$

for each $\{x, y\}$ in $S \times S$ and $g$ in $G_{2}$.

Let $O A^{\prime}$ denote the set of all members $V$ of $O A$ such that $V(x, y)$ is in $H_{2}^{\prime}$ for each $\{x, y\}$ in $S \times S$, and let $O M^{\prime}$ denote $\mathcal{E}\left(O A^{\prime}\right)$ and $\mathcal{E}^{\prime}$, the contraction of $\varepsilon$ to $O A^{\prime}$.

The Stieltjes left-integral equation. It is also the case that [3, Theorem 4.3] if $\{V, W\}$ is in $\mathcal{E}^{\prime}$, then

$$
W(x, y) g=g+(\mathrm{L}) \int_{x}^{y} W[x, I] V g
$$

for each $\{x, y\}$ in $S \times S$ and $g$ in $G_{2}$.

Reneke's nonlinear kernel functions. $F$ is the class of all functions $J$ from $S \times S$ into $H_{1}$ such that

(1) if $\{x, P\}$ is in $S \times G_{1}$, then $J(x, x) P=P$,

(2) if $\{x, P\}$ is in $S \times G_{1}$, then $J[I, x] P$ is in $G_{2}$, and

(3) there exists a member $a$ of $O A^{+}$such that if $\{t, x, y\}$ is in $S \times S \times S$ and $\{P, Q\}$ is in $G_{1} \times G_{1}$, then

$$
N_{1}([J(t, y)-J(t, x)] P-[J(t, y)-J(t, x)] Q) \leqslant a(x, y) N_{1}(P-Q) .
$$

Hinton's linear kernel functions. $F^{\prime}$ is that subset of $F$ to which $J$ belongs only in case each value of $J$ is in $H_{2}^{\prime}$.

The problem motivating much of this investigation concerns the following Stieltjes-Volterra integral equation:

$$
M_{g}(x, y)=g(x)+{ }_{y} \Sigma^{x} d J[x, I] M_{g}[L, y] .
$$

Given a function $J$ in $F$ and a function $g$ in $G_{2}$, the problem is to find a function $M_{g}$ from $S \times S$ into $G_{1}$ such that for each $\{x, y\}$ in $S \times S, M_{g}[I, y]$ is in $G_{2}$ and (3) holds.

REMARK. In expressing left (or right)-Stieltjes integrals such as in equation (3), the usual notation for such integrals (used prior to equation (3)) will be replaced by Mac Nerney's "continuously continued sum (or product)" notation [3, p. 149] used in conjunction with the left-handed choice function $L$ defined on $S \times S$ such that $L(x, y)=x$, or in conjunction with the righthanded choice function $R$ such that $R(x, y)=y$. For example, an approximating sum based on a subdivision $\left\{s_{p}\right\}_{0}^{n}$ of $\{x, y\}$, for the integral in equation (3) would be 


$$
\sum_{s} d J[x, I] M_{g}[L, y]=\sum_{p=1}^{n}\left\{J\left(x, s_{p}\right)-J\left(x, s_{p-1}\right)\right\} M_{g}\left(s_{p-1}, y\right) .
$$

Subdivisions. The statement that $t$ is a subdivision of the member $\{x, y\}$ of $S \times S$ means that $t$ is a finite sequence $\left\{t_{p}\right\}_{0}^{n}$ such that $\left\{t_{0}, t_{n}\right\}$ is $\{x, y\}$ and

(i) if $\{x, y\}$ is in $O$, then $\left\{t_{p-1}, t_{p}\right\}$ is in $O$ for each positive integer $p$ not greater than $n$, and

(ii) if $\{y, x\}$ is in $O$, then $\left\{t_{p}, t_{p-1}\right\}$ is in $O$ for each positive integer $p$ not greater than $n$.

Refinements. The statement that $r$ is a refinement of the subdivision $t$ of the member $\{x, y\}$ of $S \times S$ means that $r$ is a subdivision of $\{x, y\}$ of which $t$ is a subsequence.

Note that if $r$ refines $t$ as above, $r=\left\{r_{p}\right\}_{0}^{m}$, and $t=\left\{t_{p}\right\}_{0}^{n}$, then there exists a nondecreasing integer-valued sequence $\left\{b_{p}\right\}_{0}^{n}$ such that $b_{0}=0, b_{n}=m$, and $t$ is the composite of $r$ with $b$; i.e., $t_{p}=r\left(b_{p}\right)$ for $p=0, \ldots, n$. Such a sequence $b$ will be called a composition sequence for $t$ and $r$.

That there exists exactly one such solution $M_{g}$ for equation (3) was shown by D. B. Hinton [1, Theorem 4.1] for $J$ in $F^{\prime}$, and subsequently by J. A. Reneke [7, p. 625] for $J$ in $F$. Hinton's development of the linear theory for equation (3) concentrated on the correspondence between the kernel functions $J$, and the solution functions $M$, in the homogeneous case (when $g=1$ ). In order to address Hinton's development from within the framework of our present setting, it is necessary to construct an analogue (denoted $\mathcal{K}$ here) of Hinton's correspondence as follows.

Constant functions. For each member $P$ of $G_{1}, P$ denotes that member of $G_{2}$ whose only value is $P$.

Hinton's transformation $\mathcal{H} . \mathcal{H}$ denotes the function from $F$ such that if $J$ is in $F, \mathcal{H C}(J)$ denotes that function $M$ from $S \times S$ into $H_{1}$ such that if $\{x, y, P\}$ is in $S \times S \times G_{1}$, then $M[I, y] P$ is in $G_{2}$, and

$$
M(x, y) P=P+{ }_{y} \Sigma^{x} d J[x, I] M[L, y] P .
$$

REMARK. In the notation of equation (3), equation (4) amounts to saying that $M(x, y) P=M_{P}(x, y)$. Considering only functions $g$ which are constant corresponds to Hinton's study of the homogeneous case. The definition for $\mathcal{X}$ given above is actually more general than the linear version (described below) which was contemplated by Hinton.

The linear version of $\mathcal{H} . \mathcal{H}^{\prime}$ will denote the contraction of $\mathcal{X}$ to $F^{\prime}$.

Reneke's transformation $\Re . \Re$ denotes that function from $F$ into $O A[7$, Theorem 2] to which the ordered pair $\{J, V\}$ belongs only in case $J$ is in $F$, and

$$
\{V(x, y) g\}(t)=-{ }_{x} \Sigma^{y} d J[t, I] g[R]
$$


for each $\{x, y, t\}$ in $S \times S \times S$ and $g$ in $G_{2}$.

Reneke's main result [7, Theorem 4] was that with $\{J, V\}$ in $R,\{V, W\}$ in $\mathcal{E}$, and $g$ in $G_{2}$; then $M_{g}$ as in equation (3) is given by

$$
M_{g}(x, y)=\{W(x, y) g\}(x) .
$$

2. New foundations for the properties of $\mathcal{X}$. This section exploits the mechanism of Reneke's transformation $\Omega$ in order to trace properties of Hinton's transformation $\mathcal{X}$ to their origins in Mac Nerney's theory for the tranfsformation $\mathcal{E}$.

Theorem 2.1. Suppose $J$ is in $F$ and $V=\Re(J)$, then

$$
\text { (a) }\{V(x, y) \underline{P}\}(t)=-d J[t, I](x, y) P
$$

for each $\{x, y, t\}$ in $S \times S \times S$ and $P$ in $G_{1}$, and

$$
\text { (b) } V(x, y) g={ }_{x} \Sigma^{y} V \underline{g[R]}
$$

for each $\{x, y\}$ in $S \times S$ and $g$ in $G_{2}$, the integral existing relatively to $N_{2}$ and having for an approximating sum (over a subdivision $\left\{s_{p}\right\}_{0}^{n}$ of $\{x, y\}$ ) the member $\sum_{p=1}^{n} V\left(s_{p-1}, s_{p}\right) g\left(s_{p}\right)$ of $G_{2}$.

Proof. Suppose $\{x, y, t\}$ is in $S \times S \times S,\left\{s_{p}\right\}_{0}^{n}$ is a subdivision of $\{x, y\}$ and $P$ is in $G_{1}$; then

$$
\begin{aligned}
-\sum_{s} d J[t, I] \underline{P}[R] & =-\sum_{p=1}^{n}\left\{J\left(t, s_{p}\right) P-J\left(t, s_{p-1}\right) P\right\} \\
& =-\{J(t, y)-J(t, x)\} P=-d J[t, I](x, y) P,
\end{aligned}
$$

thus (a) is established. Now if $g$ is in $G_{2}$,

$$
\begin{aligned}
\{V(x, y) g\}(t) & =-{ }_{x} \Sigma^{y} d J[t, I] g[R]={ }_{x} \Sigma^{y}\{V \underline{g[R]}\}(t) \\
& =\left\{{ }_{x} \Sigma^{y} V \underline{g[R]}\right\}(t)
\end{aligned}
$$

hence (b). That this integral exists relatively to $N_{2}$ is equivalent with Reneke's lemma [7, p. 622].

The next theorem establishes a set of right- and left-handed integration by substitution formulae specifically tailored for this setting and motivated by the fact that all members $V$ of $R(F)$ have property (b) above.

TheOREM 2.2 (INTEGRATION BY SUBSTItUTION). Suppose $K$ is a function from $S \times S$ into $\mathrm{H}_{2}, Q$ is a function from $S$ into $\mathrm{H}_{2}$, each of $a$ and $c$ is in $\mathrm{OA}^{+}$, and for each $\{x, y, g, f\}$ in $S \times S \times G_{2} \times G_{2}$, 


$$
\begin{gathered}
N_{2}(K(x, y) g-K(x, y) f) \leqslant a(x, y) N_{2}(g-f) \text { and } \\
N_{2}(Q(y) g-Q(x) g) \leqslant c(x, y) N_{2}(g) .
\end{gathered}
$$

Suppose also that $V$ is a function from $S \times S$ such that if $\{x, y, g\}$ is in $S \times S \times G_{2}, V(x, y) g={ }_{x} \Sigma^{y} K g[R]$. Then (a) for each $\{x, y, g\}$ in $S \times S \times G_{2}$,

$$
{ }_{x} \Sigma^{y} V Q[R] g={ }_{x} \Sigma^{y} K\{\underline{Q[R] g\}[R]}
$$

provided that both integrals exist relatively to $N_{2}$. Furthermore, in case each value of $Q$ is in $H_{2}^{\prime}$, then (b) for each $\{x, y, g\}$ in $S \times S \times G_{2}$,

$$
{ }_{x} \Sigma^{y} Q[L] V g={ }_{x} \Sigma^{y} Q[L] K \underline{g[R]}
$$

provided both integrals exist relatively to $\mathrm{N}_{2}$.

LEMMA. If each of $a$ and $c$ is in $O A^{+},\{x, y\}$ is in $S \times S$, and $e$ is a positive number, then there exists a subdivision $z$ of $\{x, y\}$ such that if $\left\{s_{p}\right\}_{0}^{n}$ is a refinement of $z,\left\{r_{p}\right\}_{0}^{m}$ is a refinement of $s$, and $\left\{b_{p}\right\}_{0}^{n}$ is a composition sequence for $s$ and $r$, then

$$
\sum_{p=1}^{n} \sum_{q=b_{p-1}+1}^{b_{p}} a\left(r_{q-1}, r_{q}\right) c\left(s_{p}, r_{q}\right)<e
$$

and

$$
\sum_{p=1}^{n} \sum_{q=b_{p-1}+1}^{b_{p}} a\left(r_{q-1}, r_{q}\right) c\left(s_{p-1}, r_{q-1}\right)<e .
$$

Proof OF LemMa. There is a symmetric member $c^{\prime}$ of $O A^{+}$such that $c \leqslant c^{\prime}$ on $S \times S$. Suppose $\left\{s_{p}\right\}_{0}^{n}$ is a subdivision of $\{x, y\},\left\{r_{p}\right\}_{0}^{m}$ is a refinement of $s$, and $\left\{b_{p}\right\}_{0}^{n}$ is a composition sequence for $s$ and $r$.

$$
\begin{aligned}
\sum_{p=1}^{n} \sum_{q=b_{p-1}+1}^{b_{p}} a\left(r_{q-1}, r_{q}\right) c\left(s_{p}, r_{q}\right) & \leqslant \sum_{p=1}^{n} \sum_{q=b_{p-1}+1}^{b_{p}} a\left(r_{q-1}, r_{q}\right) c^{\prime}\left(s_{p}, r_{q}\right) \\
& =\sum_{s} a c^{\prime}[x, R]-\sum_{r} a c^{\prime}[x, R] .
\end{aligned}
$$

In a similar fashion it can be shown that

$$
\sum_{p=1}^{n} \sum_{q=b_{p-1}+1}^{b_{p}} a\left(r_{q-1}, r_{q}\right) c\left(s_{p-1}, r_{q-1}\right) \leqslant \sum_{r} a c^{\prime}[x, L]-\sum_{s} a c^{\prime}[x, L],
$$

and this is enough to establish the theorem because it is well known that the left or right integral of one nondecreasing function with respect to another exists. 
Proof of Theorem 2.2. Conclusion (a) will be established first, but for the sake of brevity, as much of the proof of conclusion (b) as is permitted without assuming each value of $Q$ to be in $H_{2}^{\prime}$ will be done in parallel. Suppose the integrals in both equations (9) and (10) exist and the hypothesis of part (a) holds.

Suppose $\{x, y\}$ is in $S \times S, g$ is in $G_{2}$, and $e>0$. There exists a subdivision $\left\{s_{p}\right\}_{0}^{n}$ of $\{x, y\}$ such that:

(1) $N_{2}\left({ }_{x} \Sigma^{y} V Q[R] g-\Sigma_{s} V Q[R] g\right)<e / 4$ and $N_{2}\left({ }_{x} \Sigma^{y} Q[L] V g-\Sigma_{s} Q[L] V g\right)<e / 4$, and

(2) if $r$ is a refinement of $s$ and $b$ is a composition sequence for $r$ and $s$, then

$$
\begin{array}{r}
N_{2}\left({ }_{x} \Sigma^{y} K \underline{\{Q[R] g\}[R]}-\sum_{r} K \underline{\{Q[R] g\}[R]}\right)<\frac{e}{4}, \\
N_{2}(g) \sum_{p=1}^{n} \sum_{q=b_{p-1}+1}^{b_{p}} a\left(r_{q-1}, r_{q}\right) c\left(s_{p}, r_{q}\right)<\frac{e}{4},
\end{array}
$$

and furthermore,

$$
\begin{aligned}
& N_{2}\left({ }_{x} \Sigma^{y} Q[L] K \underline{g[R]}-\sum_{r} Q[L] K \underline{\underline{g}[R]}\right)<\frac{e}{4}, \\
& N_{2}(g) \sum_{p=1}^{n} \sum_{q=b_{p-1}+1}^{b_{p}} a\left(r_{q-1}, r_{q}\right) c\left(s_{p-1}, r_{q-1}\right)<\frac{e}{4} .
\end{aligned}
$$

There exists a refinement $r$ of $s$ and a composition sequence $b$ for $r$ and $s$ such that if $p$ is an integer in the interval $[1, n]$, then

$$
N_{2}\left(V\left(s_{p-1}, s_{p}\right) Q\left(s_{p}\right) g-\sum_{q=b_{p-1}+1}^{b_{p}} K\left(r_{q-1}, r_{q}\right) \underline{\left.\left\{\left(s_{p}\right) g\right\}\left(r_{q}\right)\right)<\frac{e}{4 n} . ~}\right.
$$

Let

$$
A={ }_{x} \Sigma^{y} V Q[R] g-{ }_{x} \Sigma^{y} K \underline{\{Q[R] g\}[R]} .
$$

With $r$ as above,

$$
N_{2}(A)<N_{2}\left(\sum_{s} V Q[R] g-\sum_{r} K \underline{\{Q[R] g\}[R]}\right)+\frac{e}{2}<N_{2}(B)+\frac{3 e}{4},
$$

where

$$
B=\sum_{p=1}^{n} \sum_{q=b_{p-1}+1}^{b_{p}}\left[K\left(r_{q-1}, r_{q}\right) \underline{\left\{Q\left(s_{p}\right) g\right\}\left(r_{q}\right)}-K\left(r_{q-1}, r_{q}\right) \underline{\left.\left\{Q\left(r_{q}\right) g\right\}\left(r_{q}\right)\right]} .\right.
$$

Now, 


$$
\begin{aligned}
& N_{2}\left(K\left(r_{q-1}, r_{q}\right) \frac{\left\{Q\left(s_{p}\right) g\right\}\left(r_{q}\right)}{}-K\left(r_{q-1}, r_{q}\right) \underline{\left.\left\{Q\left(r_{q}\right) g\right\}\left(r_{q}\right)\right)}\right. \\
& \leqslant a\left(r_{q-1}, r_{q}\right) N_{2}\left(Q\left(s_{p}\right) g-Q\left(r_{q}\right) g\right) \leqslant a\left(r_{q-1}, r_{q}\right) c\left(s_{p}, r_{q}\right) N_{2}(g),
\end{aligned}
$$

hence

$$
N_{2}(B) \leqslant \sum_{p=1}^{n} \sum_{q=b_{p-1}+1}^{b_{p}} a\left(r_{q-1}, r_{q}\right) c\left(s_{p}, r_{q}\right) N_{2}(g)<\frac{e}{4} .
$$

Thus, this establishes the first integration by substitution equation.

To see that the second substitution equation is true, note that there exists a refinement $r$ of $s$ and a composition sequence $b$ for $r$ and $s$ such that, if $p$ is an integer in the interval $[1, n]$, then

$$
N_{2}\left(V\left(s_{p-1}, s_{p}\right) g-\sum_{q=b_{p-1}+1}^{b_{p}} K\left(r_{q-1}, r_{q}\right) g\left(r_{q}\right)\right)<\frac{e}{4 n} .
$$

Let

$$
A={ }_{x} \Sigma^{y} Q[L] V g-{ }_{x} \Sigma^{y} Q[L] K \underline{g[R]} .
$$

With $r$ as above,

$$
N_{2}(A)<N_{2}\left(\sum_{s} Q[L] V g-\sum_{r} Q[L] K \underline{g[R]}\right)+\frac{e}{2}<N_{2}(B)+\frac{3 e}{4},
$$

where

$$
\begin{aligned}
B=\sum_{p=1}^{n} Q\left(s_{p-1}\right) \sum_{q=b_{p-1}+1}^{b_{p}} K\left(r_{q-1}, r_{q}\right) \underline{g\left(r_{q}\right)} \\
-\sum_{p=1}^{n} \sum_{q=b_{p-1}+1}^{b_{p}} Q\left(r_{q-1}\right) K\left(r_{q-1}, r_{q}\right) g\left(r_{q}\right) .
\end{aligned}
$$

Because of the assumed property of $Q$ under the hypothesis for part (b),

$$
\begin{gathered}
N_{2}\left(Q\left(s_{p-1}\right) \sum_{q=b_{p-1}+1}^{b_{p}} K\left(r_{q-1}, r_{q}\right) \underline{g\left(r_{q}\right)}-\sum_{q=b_{p-1}+1}^{b_{p}} Q\left(r_{q-1}\right) K\left(r_{q-1}, r_{q}\right) g\left(r_{q}\right)\right) \\
\leqslant \sum_{q=b_{p-1}+1}^{b_{p}} c\left(s_{p-1}, r_{q-1}\right) N_{2}\left(K\left(r_{q-1}, r_{q}\right) \underline{\left.g\left(r_{q}\right)\right)}\right. \\
\leqslant \sum_{q=b_{p-1}+1}^{b_{p}} c\left(s_{p-1}, r_{q-1}\right) a\left(r_{q-1}, r_{q}\right) N_{2}(g) .
\end{gathered}
$$


Thus $N_{2}(B)<e / 4$ and the second substitution equation is established.

The next theorem, which arises from an application of equation (9), is a generalization of Reneke's main result [7, Theorem 4, p. 624].

THEOREM 2.3. If $\{V, W\}$ is in $\varepsilon$ and for each $\{x, y, g\}$ in $S \times S \times G_{2}, V(x, y) g$ $={ }_{x} \Sigma^{y} V \underline{g[R]}$, then

$$
W(x, y) g=g+{ }_{x} \Sigma^{y} V \underline{\{W[R, y] g\}[R]}
$$

for each $\{x, y, g\}$ in $S \times S \times G_{2}$.

Proof. With $V$ and $W$ as in the hypothesis, let $t$ denote a member of $S$ and make the identifications $Q=W[I, t]$ and $K=V$. That the hypothesis of Theorem 2.2 is satisfied relatively to $V$ and $K$ is a consequence of Theorem 2.1 ; that it is satisfied relatively to $Q$ is a consequence of [6, Lemma $2.1, \mathrm{p}$. 627]. Thus for each $\{x, y, g\}$ in $S \times S \times G_{2}$,

$$
{ }_{x} \Sigma^{y} V W[R, t] g={ }_{x} \Sigma^{y} V \underline{\{W[R, t] g\}[R]} .
$$

Recalling the Stieltjes right-integral equation mentioned earlier, and specializing $t$ to be $y$ in the above yields,

$$
W(x, y) g=g+{ }_{x} \Sigma^{y} V W[R, y] g=g+{ }_{x} \Sigma^{y} V \underline{\{W[R, y] g\}[R]} .
$$

REMark. The way in which Theorem 2.3 includes Reneke's main result may be indicated as follows: Suppose that $J$ is in $F,\{J, V\}$ is in $\Re$, and $\{V, W\}$ is in E; then by Theorems 2.1 and 2.3 we have equation (11) for all $\{x, y, g\}$ in $S \times S \times G_{2}$. Now if equation (11) is evaluated at a member $t$ of $S$, then by equation (7) and a change from right to left integrals, there results:

$$
\{W(x, y) g\}(t)=g(t)+{ }_{y} \Sigma^{x} d J[t, I]\{W[L, y] g\}[L] .
$$

Further specialization of $t$ to be $x$ yields Reneke's main result:

$$
\{W(x, y) g\}(x)=g(x)+{ }_{y} \Sigma^{x} d J[x, I]\{W[L, y] g\}[L],
$$

so that with $M_{g}(x, y)=\{W(x, y) g\}(x)$ for each $\{x, y\}$ in $S \times S$, equation (3) holds.

Turning our attention now to the linear situation $\left(J\right.$ in $\left.F^{\prime}\right)$, an application of equation (10) yields the following generalization of Hinton's Theorem 4.1 [1, p. 325].

THEOREM 2.4. If $\{V, W\}$ is in $\mathcal{E}^{\prime}$, then

$$
{ }_{x} \Sigma^{y} W[x, L] V \underline{g[R]}={ }_{x} \Sigma^{y} d W[x, I] \underline{g[R]}
$$


for each $\{x, y, g\}$ in $S \times S \times G_{2}$, and (b) if additionally,

$$
V(x, y) g={ }_{x} \Sigma^{y} V \underline{g[R]}
$$

for each $\{x, y, g\}$ in $S \times S \times G_{2}$, then for each $\{x, y, g\}$ in $S \times S \times G_{2}$,

$$
W(x, y) g-g={ }_{x} \Sigma^{y} d W[x, I] \underline{g[R]} .
$$

Proof. Since $\{V, W\}$ is in $\mathcal{E}$, there exists a member $\{a, m\}$ of $\mathcal{E}^{+}$such that $\Sigma\{m-1\}=a$ and

$$
N_{2}([W(x, y)-1-V(x, y)] g) \leqslant\{m(x, y)-1-a(x, y)\} N_{2}(g)
$$

for each $\{x, y, g\}$ in $S \times S \times G_{2}$. Thus one can see that if $s$ is a subdivision of the member $\{x, y\}$ of $S \times S$ and $g$ is in $G_{2}$, then

$$
\begin{aligned}
N_{2}\left(\sum_{s} W[x, L] V \underline{g[R]}-\sum_{s} d W[x, I] \underline{g[R]}\right) \\
\quad=N_{2}\left(\sum_{s} W[x, L] V \underline{g[R]}-\sum_{s} W[x, L]\{W-1\} \underline{g[R]}\right) \\
\quad=N_{2}\left(\sum_{s} W[x, L]\{W-1-V\} \underline{g[R]}\right) \leqslant m(x, y) N_{2}(g) \sum_{s}\{m-1-a\} .
\end{aligned}
$$

Therefore if $\{x, y, g\}$ is in $S \times S \times G_{2}$,

$$
{ }_{x} \Sigma^{y} W[x, L] V \underline{g[R]}={ }_{x} \Sigma^{y} d W[x, I] \underline{g[R]}
$$

and an application of equation (10) to the Stieltjes left-integral equation yields:

$$
\begin{aligned}
W(x, y) g & =g+{ }_{x} \Sigma^{y} W[x, L] V g=g+{ }_{x} \Sigma^{y} W[x, L] V \underline{g[R]} \\
& =g+{ }_{x} \Sigma^{y} d W[x, I] \underline{g[R]} .
\end{aligned}
$$

REMARK. That the preceding result implies Hinton's Theorem 4.1 may be seen as follows: Suppose $J$ is in $F^{\prime},\{J, V\}$ is in $\mathscr{R}$, and $\{V, W\}$ is in $\varepsilon$. Note that $\{V, W\}$ is in $\mathcal{E}^{\prime}$ and that by Theorems 2.1 and 2.4, we have equation (13) for all $\{x, y, g\}$ in $S \times S \times G_{2}$. Now, evaluation at a member $t$ of $S$, of equation (13) yields

$$
\{W(x, y) g\}(t)=g(t)+{ }_{x} \Sigma^{y}\{d W[x, I] \underline{g[R]}\}(t),
$$

and if $t=x$, and $M$ denotes $\mathscr{T}(J)$, then

$$
\{W(x, y) g\}(x)=g(x)+{ }_{x} \Sigma^{y} d M[x, I] g[R] .
$$


In the notation of equation (3) this becomes

$$
M_{g}(x, y)=g(x)+{ }_{x} \Sigma^{y} d M[x, I] g[R],
$$

which is Hinton's Theorem $4.1[1, \mathrm{p}$. 325] relating the solutions of the nonhomogeneous equation (3) to the solutions of the homogeneous version of equation (3).

REMARK. Equation (14) affords an opportunity to define the $M_{g}$ notation which has been used somewhat loosely thus far.

Definition. Suppose $Z$ is a function from $S \times S$ into $H_{1}$ such that if $t$ is in $S$, there exists a member $a$ of $O A^{+}$such that for each $\{x, y, P, Q\}$ in $S \times S \times G_{1} \times G_{1}$,

$$
N_{1}([Z(t, y)-Z(t, x)] P-[Z(t, y)-Z(t, x)] Q) \leqslant a(x, y) N_{1}(P-Q) .
$$

If $g$ is in $G_{2}, Z_{g}$ denotes that function from $S \times S$ given by

$$
Z_{g}(x, y)=g(x)+{ }_{x} \Sigma^{y} d Z[x, I] g[R]
$$

for each $\{x, y\}$ in $S \times S$ (the conditions on $Z$ mentioned above being sufficient to guarantee the existence of the integral involved).

The next theorem presents a new proof of Hinton's Theorem 3.3 [1, p. 324] which states, in our notation, that $\mathcal{H}^{\prime}=\left(\mathcal{C}^{\prime}\right)^{-1}$.

THEOREM 2.5. If $\{J, M\}$ is in ' $\mathcal{C}^{\prime}$, then $\{M, J\}$ is in $\mathcal{I C}^{\prime}$.

Proof. Suppose $\{J, M\}$ is in $\mathcal{X}^{\prime}$. Then for each $\{x, y, g\}$ in $S \times S \times G_{2}$,

$$
M_{g}(x, y)=g(x)+{ }_{x} \Sigma^{y} d M[x, I] g[R] .
$$

Pick a member $P$ of $G_{1}$ and, regarding $y$ as fixed, take $f=J[I, y] P$. Since $f$ is in $G_{2}$,

$$
M_{f}(x, y)=J(x, y) P+{ }_{x} \Sigma^{y} d M[x, I] J[R, y] P
$$

hence

$$
J(x, y) P=M_{f}(x, y)+{ }_{y} \Sigma^{x} d M[x, I] J[L, y] P
$$

for each $x$ in $S$. Now (since $P$ and $y$ are arbitrary), all that remains to be done in order to show that $J=\mathcal{K}^{\prime}(M)$ is to show that $M_{f}[I, y]=P$. Toward this end, let $h=M_{f}[I, y]$. By the remark following Theorem 2.3 we know that

$$
h(x)=J(x, y) P+{ }_{y} \Sigma^{x} d J[x, I] h[L]
$$

for each $x$ in $S$. However, it is easy to see that $P$, just as $h$, also solves this integral equation. Since $h\left[7\right.$, Theorem 3, p. 624] and $P$ are both in $G_{2}$, and the 
integral equation above has only one solution in $G_{2}$, it follows that $h=P$. We may conclude that

$$
J(x, y) P=P+{ }_{y} \Sigma^{x} d M[x, I] J[L, y] P
$$

for each $\{x, y, P\}$ in $S \times S \times G_{1}$, so that $\{M, J\}$ is in $\mathcal{X}^{\prime}$.

Thus we see that via the proof of Theorem 2.4, the fact that $\mathcal{S C}^{\prime}=\left(\mathcal{S C}^{\prime}\right)^{-1}$ stems from the left-Stieltjes integral equation (equation (2)) for members $\{V, W\}$ of $\mathcal{E}^{\prime}$.

3. A characterization and other results. Preliminary to providing a complete characterization of the sets $\mathscr{R}\left(F^{\prime}\right)$ and $\mathcal{E}\left(\mathcal{R}\left(F^{\prime}\right)\right)$, it is convenient to observe that the transformation $\mathscr{E}$ sets up a reversible correspondence between two subsets of $O A^{\prime}$ and $O M^{\prime}$, respectively, as distinguished in the following theorem.

THEOREM 3.1. If $\{V, W\}$ is in $\mathcal{E}^{\prime}$, the following are equivalent:

(i) $V(x, y) g={ }_{x} \Sigma^{y} V g[R]$ for each $\{x, y, g\}$ in $S \times S \times G_{2}$, and

(ii) $W(x, y) g-g={ }_{x} \Sigma^{y} d W[x, I] g[R]$ for each $\{x, y, g\}$ in $S \times S \times G_{2}$.

Proof. That (i) implies (ii) was shown in Theorem 2.4. To see that (ii) implies (i), one should recall the fact that

$$
V(x, y) g={ }_{x} \Sigma^{y}[W-1] g \text { for each }\{x, y, g\} \text { in } S \times S \times G_{2},
$$

and that by hypothesis and part (a) of Theorem 2.4,

$$
W(x, y) g-g={ }_{x} \Sigma^{y} d W[x, I] \underline{g[R]}={ }_{x} \Sigma^{y} W[x, L] V \underline{g[R]}
$$

for each $\{x, y, g\}$ in $S \times S \times G_{2}$. Let $\{a, m\}$ denote a member of $\mathcal{E}^{+}$such that $N_{2}(V g) \leqslant a N_{2}(g)$ and $N_{2}(W g-g) \leqslant\{m-1\} N_{2}(g)$ for each $g$ in $G_{2}$. Suppose $\{x, y\}$ is in $S \times S,\left\{s_{p}\right\}_{0}^{n}$ is a subdivision of $\{x, y\}$ and $e$ is a positive number. There exists a refinement $r$ of $s$ such that if $b$ is a composition sequence for $s$ and $r$, then

$$
N_{2}\left(W\left(s_{p-1}, s_{p}\right) g-g-\sum_{q=b_{p-1}+1}^{b_{p}} W\left(s_{p-1}, r_{q-1}\right) V\left(r_{q-1}, r_{q}\right) g\left(r_{q}\right)\right)<\frac{e}{n}
$$

for each $p$ in $[1, n]$, and

$$
\sum_{p=1}^{n} \sum_{q=b_{p-1}+1}^{b_{p}} d m[x, I]\left(s_{p-1}, r_{q-1}\right) a\left(r_{q-1}, r_{q}\right)<\frac{e}{\left\{1+N_{2}(g)\right\}} .
$$

Let 


$$
\begin{aligned}
T_{s}= & \sum_{s}[W-1] g-\sum_{r} V \underline{g[R]} \\
= & \sum_{p=1}^{n}\left[W\left(s_{p-1}, s_{p}\right) g-g-\sum_{q=b_{p-1}+1}^{b_{p}} W\left(s_{p-1}, r_{q-1}\right) V\left(r_{q-1}, r_{q}\right) \underline{g\left(r_{q}\right)}\right] \\
& +\sum_{p=1}^{n} \sum_{q=b_{p-1}+1}^{b_{p}}\left[W\left(s_{p-1}, r_{q-1}\right) V\left(r_{q-1}, r_{q}\right) \underline{g\left(r_{q}\right)}-V\left(r_{q-1}, r_{q}\right) \underline{g\left(r_{q}\right)}\right],
\end{aligned}
$$

so that,

$$
\begin{aligned}
N_{2}\left(T_{s}\right) & \leqslant \sum_{p=1}^{n} \frac{e}{n}+\sum_{p=1}^{n} \sum_{q=b_{p-1}+1}^{b_{p}}\left\{m\left(s_{p-1}, r_{q-1}\right)-1\right\} a\left(r_{q-1}, r_{q}\right) N_{2}(g) \\
& <e+N_{2}(g) \sum_{p=1}^{n} \sum_{q=b_{p-1}+1}^{b_{p}} m\left(x, s_{p-1}\right)^{-1}\left\{m\left(x, r_{q-1}\right)-m\left(x, s_{p-1}\right)\right\} a\left(r_{q-1}, r_{q}\right) \\
& <2 e
\end{aligned}
$$

hence

$$
V(x, y) g={ }_{x} \Sigma^{y}[W-1] g={ }_{x} \Sigma^{y} V \underline{g[R]} .
$$

REMARK. Both part (a) of Theorem 2.4 and the present implication (ii) $\rightarrow$ (i) are also valid for $\{V, W\}$ in $\&$, i.e. in the nonlinear setting.

The next theorem completely characterizes the sets $\Re\left(F^{\prime}\right)$ and $\mathcal{E}^{\prime}\left(\Re\left(F^{\prime}\right)\right)$.

THEOREM 3.2. Suppose $\{V, W\}$ is in $\mathcal{G}^{\prime}$. In order that there exist a member $J$ of $F^{\prime}$ such that $V=\mathscr{R}(J)$, it is necessary and sufficient that (a) for each $P$ in $G_{1}$, $V \underline{P}$ is fully additive (i.e. $V(x, t) \underline{P}+V(t, y) \underline{P}=V(x, y) P$ for each $\{t, x, y\}$ in $S \times S \times S$ ), and (b) either condition (i) or condition (ii) of Theorem 3.1 holds. Furthermore, $J(x, y) P=P-\{V(x, y) P\}(x)$ for each $\{x, y, P\}$ in $S \times S \times G_{1}$.

Proof. Under the hypothesis above, let $J$ be defined as shown above and recall that by Theorem 3.1 we are assured that condition (i) of that theorem holds. Since $V$ is fully additive on the constant members of $G_{2}$, a straightforward calculation will show that for each $\{x, y, t, P\}$ in $S \times S \times S \times G_{1}$,

$$
-d J[t, I](x, y) P=\{V(x, y) P\}(t) .
$$

Hence one can easily see that $J$ is in $F^{\prime}$ and $\{V(x, y) g\}(t)={ }_{x} \Sigma^{y}\{V g[R]\}(t)$ $=-{ }_{x} \Sigma^{y} d J[t, I] g[R]$ for each $\{x, y, t, g\}$ in $S \times S \times S \times G_{2}$. Thus $V=\Re(J)$, so that sufficiency is established. The necessity of the conditions may be easily seen from Theorems 2.1 and 2.4 and some simple calculations stemming from the required assumption that $V=\mathscr{R}(J)$. 
THEOREM 3.3. If $\{V, W\}$ is in $\mathcal{E}^{\prime}$, condition (i) of Theorem 3.1 holds, and each of $A$ and $B$ is a function $D$ from $S \times S$ into $G_{1}$ such that for each $y$ in $S, D[I, y]$ is in $G_{2}$, then the following are equivalent:

(1) $B(x, y)=A(x, y)+{ }_{x} \Sigma^{y}\{V B[R, y]\}(x)$ for each $\{x, y\}$ in $S \times S$, and

(2) $A(x, y)=B(x, y)-{ }_{x} \Sigma^{y}\{d W[x, I] A[R, y]\}(x)$ for each $\{x, y\}$ in $S \times S$.

Proof. (1) implies (2): Assume the hypothesis and condition (1). Let $\tilde{B}$ denote a function from $S \times S$ into $G_{1}$ such that

$$
\tilde{B}(x, y)=\{W(x, y) A[I, y]\}(x) \text { for each }\{x, y\} \text { in } S \times S .
$$

By Theorem 2.3 it follows that

$$
\tilde{B}(x, y)=A(x, y)+{ }_{x} \Sigma^{y}\{V \underline{\tilde{B}[R, y]}\}(x) \text { for each }\{x, y\} \text { in } S \times S,
$$

so that $\tilde{B}$, just as $B$, solves the integral equation of conditon (1). Therefore $\tilde{B}=B$. Now, by Theorem 3.1 we have

$$
B(x, y)=\{W(x, y) A[I, y]\}(x)=A(x, y)+{ }_{x} \Sigma^{y}\{d W[x, I] A[R, y]\}(x),
$$

which establishes (2).

(2) implies (1): Assume condition (2) holds. Using Theorem 3.1, we may observe that for each $\{x, y\}$ in $S \times S$,

$$
\begin{aligned}
B(x, y) & =A(x, y)+{ }_{x} \Sigma^{y}\{d W[x, I] A[R, y]\}(x) \\
& =A(x, y)+\{W(x, y) A[I, y]\}(x)-A(x, y) .
\end{aligned}
$$

However, by Theorem 2.3, the fact that $B(x, y)=\{W(x, y) A[I, y]\}(x)$ for each $\{x, y\}$ in $S \times S$ implies that for each $\{x, y\}$ in $S \times S$,

$$
B(x, y)=A(x, y)+{ }_{x} \Sigma^{y}\{V \underline{B[R, y]}\}(x) .
$$

COROLLARY. If $\{J, M\}$ is in $\mathcal{I}^{\prime}$ and each of $A$ and $B$ is a function $D$ from $S \times S$ into $G_{1}$ such that for each $y$ in $S, D[I, y]$ is in $G_{2}$, then the following are equivalent:

(i) $B(x, y)=A(x, y)+{ }_{y} \Sigma^{x} d J[x, I] B[L, y]$ for each $\{x, y\}$ in $S \times S$, and

(ii) $A(x, y)=B(x, y)+{ }_{y} \Sigma^{x} d M[x, I] A[L, y]$ for each $\{x, y\}$ in $S \times S$.

This corollary has an easy interpretation in terms of input-output functions for hereditary systems similar to those treated by Reneke [8]. Suppose we regard the kernel function $J$ together with the integral equation (i) above as defining an hereditary system $H_{J}$ which acts upon input functions such as $A$ in order to produce output functions such as $B$; i.e. $B=H_{J}(A)$. We see that with $M=\mathscr{I C}^{\prime}(J)$, the hereditary system $H_{M}$ is the inverse of the system $H_{j}$; i.e. $B=H_{J}(A)$ only in case $A=H_{M}(B)$. 
Conclusion. We have shown that a right-handed, nonlinear integration by substitution process is an essential feature of Reneke's solution technique and have used that process to refine and extend Reneke's principle result. In the linear setting, a left-handed integration by substitution process has been isolated and used to completely characterize the sets $\Re\left(F^{\prime}\right)$ and $\mathcal{E}\left(\Re\left(F^{\prime}\right)\right)$. Hinton's result, $\mathcal{I C}^{\prime}=\left(\mathcal{C C}^{\prime}\right)^{-1}$, has been shown to be a consequence of the fact that $\mathcal{E}^{\prime}$ provides solutions to the linear Stieltjes left-integral equation. This study has been, in a sense, a continuation of Reneke's effort to refine and extend the theory for Stieltjes-Volterra integral equations by utilizing the properties of the transformation $\mathcal{E}$. For another example in which application of the transformation $\mathcal{E}$ has resulted in the extension of existing theory, the reader is referred to a recent paper [2] by A. J. Kay in which certain results of B. W. Helton are extended to a nonlinear setting.

\section{BIBLIOGRAPHY}

1. D. B. Hinton, A Stieltjes-Volterra integral equation theory, Canad. J. Math. 18 (1966), 314-331. MR 32 \#6169.

2. A. J. Kay, Nonlinear integral equations and product integrals, Pacific J. Math. 60 (1975), 203-222.

3. J. S. Mac Nerney, Integral equations and semigroups, Illinois J. Math. 7 (1963), 148-173. MR 26 \# 1726.

4. -, A linear initial-value problem, Bull. Amer. Math. Soc. 69 (1963), 314-329. MR 26 \#4133.

5. - Note on successive approximations, Rend. Circ. Mat. Palermo (2) 12 (1963), 87-90. MR 29 \#3867.

6. - A nonlinear integral operation, Illinois J. Math. 8 (1964), 621-638. MR 29 \# 5082.

7. J. A. Reneke, A product integral solution of a Stieltjes-Volterra integral equation, Proc. Amer. Math. Soc. 24 (1970), 621-626. MR 40 \#6214.

8. - Product integral solutions for hereditary systems, Trans. Amer. Math. Soc. 181 (1973), 483-493. MR 48 \#2692.

16210 La Avenida Drrve, Houston, Texas 77062 